PROCEEDINGS OF THE

AMERICAN MATHEMATICAL SOCIETY

Volume 135, Number 10, October 2007, Pages 3061-3071

S $0002-9939(07) 08853-3$

Article electronically published on May 14, 2007

\title{
PRODUCT RULE WINS A COMPETITIVE GAME
}

\author{
ANDREW BEVERIDGE, TOM BOHMAN, ALAN FRIEZE, AND OLEG PIKHURKO
}

(Communicated by Jim Haglund)

\begin{abstract}
We consider a game that can be viewed as a random graph process. The game has two players and begins with the empty graph on vertex set $[n]$. During each turn a pair of random edges is generated and one of the players chooses one of these edges to be an edge in the graph. Thus the players guide the evolution of the graph as the game is played. One player controls the even rounds with the goal of creating a so-called giant component as quickly as possible. The other player controls the odd rounds and has the goal of keeping the giant from forming for as long as possible. We show that the product rule is an asymptotically optimal strategy for both players.
\end{abstract}

\section{INTRODUCTION}

Consider the following random graph game. There are two players, Creator and Destroyer, and the game is played on vertex set $[n]$. The game begins with the empty graph, which we denote $G_{0}$. The game is played in a sequence of turns that alternate between Creator and Destroyer. During turn $i$ a pair of random edges $e_{i}, f_{i}$ is generated and the player who controls round $i$ chooses one of these edges to be an edge in the graph, adding it to $G_{i-1}$ to form the graph $G_{i}$. We define a giant component to be any connected component with at least $n / \log n$ vertices. (A giant component is usually defined as a component with $\Omega(n)$ vertices. We choose a threshold of $n / \log n$ here in order to define the game without reference to asymptotics in $n$. When we state our formal results, we will take $n \rightarrow \infty$, and it will turn out that our choice here is somewhat arbitrary.) Creator's goal is to achieve the formation of a giant component in $G_{i}$ as quickly as possible while Destroyer's goal is to keep a giant from forming for as long as possible. One way to measure the success of the players is to compare with the phase transition in the Erdős-Rényi random graph; that is, we might say that if $G_{c n}$ has a giant where $c$ is a constant less than $1 / 2$, then Creator wins and if $G_{c n}$ does not have a giant where $c$ is a constant greater than $1 / 2$, then Destroyer wins.

If we fix the strategies for the two players, then this game gives us an example of an Achlioptas process, a random graph process in which we choose one edge from each pair in a sequence $e_{1}, f_{1} ; e_{2}, f_{2} ; \ldots$ of pairs of random edges where the choices are determined by some fixed algorithm and are made on-line. For discussion

Received by the editors February 1, 2006 and, in revised form, June 15, 2006.

2000 Mathematics Subject Classification. Primary 05C80.

The second author was supported in part by NSF grant DMS-0401147.

The third author was supported in part by NSF grant CCR-0502793.

The fourth author was supported in part by NSF grant DMS-0457512.

(C)2007 American Mathematical Society 
of Achlioptas processes and results on the timing of the emergence of the giant component in these and related models see [1, [2, 3], 4], [5] and [7].

Let $G$ be a fixed graph with connected components $C_{1}, \ldots, C_{r}$. We define the susceptibility of $G$ to be

$$
X(G)=\frac{1}{n} \sum_{i=1}^{r}\left|C_{i}\right|^{2}
$$

where $\left|C_{i}\right|$ is the number of vertices in component $C_{i}$. Note that the susceptibility gives the expected number of vertices in the connected component containing a vertex chosen uniformly at random. This parameter plays an important role in percolation theory. Motivated (in part) by this connection, Spencer introduced the product rule, which is the Achlioptas process that chooses the edge that minimizes the change in the susceptibility [6]. Note that the change in the susceptibility when we add to $G$ an edge $e$ that connects distinct components $C_{i}$ and $C_{j}$ is $\left(2 \cdot\left|C_{i}\right| \cdot\left|C_{j}\right|\right) / n$. Thus to minimize the increase in the susceptibility we choose the edge from the pair $e_{i}, f_{i}$ that minimizes the product of the sizes of the components joined. This (together with an arbitrary convention for breaking ties and dealing with edges that fall within a single component) defines the product rule. It is believed that the product rule is a nearly optimal Achlioptas process with respect to delaying the appearance of the giant component for as long as possible (for discussion of this issue and some examples of Achlioptas processes that appear to do slightly better than the product rule see [7]). Very little is known about the graph evolution given by the product rule; for example, the timing and nature of the appearance of a giant component in the product rule are intriguing open issues.

In order to state our results on the game defined above, we must make a distinction between the product rule that maximizes the change in the susceptibility and the product rule as defined above, which minimizes the change in susceptibility. Suppose $e_{i}, f_{i}$ is a pair of random edges and $G_{i-1}$ is a fixed graph. Suppose further that $e_{i}$ connects components in $G_{i-1}$ consisting of $a$ and $b$ vertices, respectively, while $f_{i}$ connects components consisting of $c$ and $d$ vertices, respectively. Destroyer's product rule is defined by

$$
\text { Destroyer's product rule chooses } \begin{cases}e_{i} & \text { if } a b<c d \\ f_{i} & \text { if } a b>c d\end{cases}
$$

(Note that Destroyer's product rule is identical to the product rule defined above.) Analogously, we define Creator's product rule by stipulating that

$$
\text { Creator's product rule chooses } \begin{cases}f_{i} & \text { if } a b<c d \\ e_{i} & \text { if } a b>c d\end{cases}
$$

For both of these rules ties (i.e., $a b=c d$ ) and edges that fall within a single component are handled arbitrarily. We assume throughout that edges are chosen uniformly and independently at random from $[n]^{2}$. Of course, this convention will lead to the appearance of loops and multiple edges in our process. As the number of these is bounded by, say, $\log n \mathbf{w h p}$, they can be removed from the process without altering our results.

Theorem 1.1. Let $G_{i}$ be the graph produced through $i$ rounds of our competitive game. 
(a) If Destroyer employs Destroyer's product rule and $c<1 / 2$, then whp the largest component of $G_{c n}$ has $O(\log n)$ vertices.

(b) If Creator employs Creator's product rule and $c>1 / 2$, then whp the largest component of $G_{c n}$ has $\Omega(n)$ vertices.

We prove this theorem by applying techniques developed by Spencer and Wormald in a proof that the evolution of the susceptibility determines the timing of the phase transition for a certain natural class of Achlioptas processes. (This result was independently proved by Bohman and Kravitz [4, and the methods developed in [4 can also be used to prove Theorem 1.1. However, the bound on the component sizes in part (a) would not be as strong, so we opt for the methods of Spencer and Wormald here.)

Suppose we measure success in our competitive game relative to the Erdős-Rényi phase transition. Our methods can be used to show not only that the product rule does not lose the game (as we saw in Theorem 1.1) but also that the product rule wins when the other player uses a strategy that varies significantly from the product rule.

Theorem 1.2. Let $G_{i}$ be the graph produced through $i$ rounds of our competitive game. Let the random variables $A_{i}$ and $B_{i}$ be the number of times Creator and Destroyer, respectively, do not follow their respective product rules during the first $i$ turns of the game. Let $\epsilon>0$ be a constant.

(a) If Destroyer employs Destroyer's product rule and Creator uses a rule for which $A_{n / 2}>\epsilon n \mathbf{w h p}$, then there exists a constant $c>1 / 2$ such that whp the largest component of $G_{c n}$ has $O(\log n)$ vertices.

(b) If Creator employs Creator's product rule and Destroyer uses a rule for which $B_{n / 2}>\epsilon n \mathbf{w h p}$, then there exists a constant $c<1 / 2$ such that $\mathbf{w h p}$ the largest component of $G_{c n}$ has $\Omega(n)$ vertices.

Theorem 1.2 implies, for example, that the phase transition for the product rule (i.e., we apply Destroyer's product rule in every round) occurs after $(1 / 2+\delta) n$ edges for some fixed $\delta>0$. Furthermore, Theorem 1.2 can be used to analyze the following variation on Achlioptas processes. Suppose that there is one player who simultaneously builds two graphs $G$ and $H$ on the same vertex set. As before, two random edges arrive in each round. The player has to decide on-line which edge is added to $G$, while the other edge goes to $H$. The objective of the player is to control the birth of the giant in both graphs; for example, to delay it in $G$ and to create it in $H$. It follows from Theorem 1.2 that whp the player can strictly beat the Erdös-Rényi threshold in both graphs. In the example given, the player uses Destroyer's product rule with respect to $G$ in odd rounds and Creator's product rule with respect to $H$ in even rounds. To see that 'mistakes' are made (by an imaginary opponent) in the formation of both $G$ and $H$, consider the set of vertices $X$ that are isolated in both $G$ and in $H$ and the set of vertices $Y$ that are isolated in neither $G$ nor $H$. Both $X$ and $Y$ are a positive proportion of the vertices from, say, step $n / 100$ until step $n / 2$. Therefore, there will be a positive proportion of rounds when $e_{i}$ lies in $X$ and $f_{i}$ lies in $Y$. In such rounds the player is making the correct choice in both graphs. 


\section{Preliminaries}

We begin with some background (taken from [6] and [7]). The starting point for our analysis is a differential equation that describes the evolution of the susceptibility in the Erdős-Rényi random graph. Suppose $G$ is a fixed graph on vertex set $[n]$ with connected components $C_{1}, \ldots, C_{r}$. Let $e$ be a random edge, and set $G^{+}=G+e$. If $e$ connects components $C_{i}$ and $C_{j}$, then the change in the susceptibility is $\left(2 \cdot\left|C_{i}\right| \cdot\left|C_{j}\right|\right) / n$. Thus

$$
\begin{aligned}
& E\left[X\left(G^{+}\right)-X(G)\right]=\sum_{i \neq j} \frac{\left|C_{i}\right|\left|C_{j}\right|}{n^{2}} \frac{2\left|C_{i}\right|\left|C_{j}\right|}{n} \\
&=\frac{2}{n}\left(\sum_{i=1}^{r} \frac{\left|C_{i}\right|^{2}}{n}\right)^{2}-2 \sum_{i=1}^{r} \frac{\left|C_{i}\right|^{4}}{n^{3}}=\frac{2 X^{2}(G)}{n}-2 \sum_{i=1}^{r} \frac{\left|C_{i}\right|^{4}}{n^{3}} .
\end{aligned}
$$

Since the sum of the fourth powers of the component sizes divided by $n^{3}$ is negligible when all components are small, we expect the susceptibility of the Erdös-Rényi random graph $G_{n, c n}$ to be concentrated around $f(c)$ where $f$ is the solution to the differential equation

$$
f^{\prime}=2 f^{2}, \quad f(0)=1 .
$$

Note that

$$
f(x)=\frac{1}{1-2 x} .
$$

This gives a nice explanation for the fact that the giant component suddenly appears in the Erdős-Rényi graph when there are about $n / 2$ random edges. (i.e., at about $n / 2$ random edges the susceptibility blows up, and this should reflect a dramatic change in the component structure.) Of course, this is a formal proof of neither the concentration of the susceptibility around this trajectory nor the sudden emergence of the giant. See Bohman and Kravitz [4] or Spencer and Wormald [7] for proper developments along these lines.

A key piece of the Spencer and Wormald proof is the following definition and theorem. For a vertex $v$ of graph $G$ we let $C_{v}$ be the connected component of $G$ that contains $v$. We say that $G$ has a $K, \alpha$ component tail if

$$
\frac{1}{n}\left|\left\{v:\left|C_{v}\right| \geq s\right\}\right| \leq K e^{-\alpha s} \quad \text { for all } s .
$$

Note that if $G$ has a $K, \alpha$ component tail and $\alpha \alpha^{\prime}>1$, then

$$
\max _{v}\left|C_{v}\right|<\alpha^{\prime} \log n
$$

for $n$ sufficiently large. The random graph $G_{n, p}$ is the random graph on vertex set $[n]$ in which each of the $\left(\begin{array}{l}n \\ 2\end{array}\right)$ possible edges appears independently with probability $p$.

Theorem 2.1 (Spencer, Wormald). Let $L, K, \alpha>0$ be constants. Let $G$ be a graph on $n$ vertices with a $K, \alpha$ component tail . Let $H$ be the random graph $H=G_{n, d / n}$ where $d$ is a fixed constant. Set $G^{+}=G \cup H$.

(a) If $d L<1$ and $X(G)<L$, then there exist $K^{+}, \alpha^{+}$such that whp $G^{+}$has a $K^{+}, \alpha^{+}$component tail.

(b) If $d L>1$ and $X(G)>L$, then whp $G^{+}$has a component with $\Omega(n)$ vertices. 


\section{Proof of Theorem 1.1}

Our key observation is that if the product rule is employed (by either player), then the expected change in the susceptibility in two turns of the game is bounded by the expected change in the susceptibility of the Erdős-Rényi random graph when two random edges are added.

Lemma 3.1. Let $G$ be a fixed graph on vertex set $[n]$ with components $C_{1}, \ldots, C_{r}$. Let $e_{1}, f_{1}, e_{2}, f_{2} \in[n]^{2}$ be independent random edges. If $G^{+}=G+\{a, b\}$ where $a \in\left\{e_{1}, f_{1}\right\}$ is chosen according to Destroyer's product rule and $b \in\left\{e_{2}, f_{2}\right\}$ is chosen arbitrarily, then

$$
E\left[X\left(G^{+}\right)\right] \leq X(G)+\frac{4}{n} X^{2}(G)+\frac{32}{n^{2}} X^{3}(G) .
$$

Proof. For each edge $e \in[n]^{2}$ set $\Delta_{e}=2 \cdot\left|C_{1}\right| \cdot\left|C_{2}\right|$ if $e$ joins distinct components $C_{1}, C_{2}$ of $G$ and set $\Delta_{e}=0$ if $e$ lies within a connected component. For each positive integer $m$ let $p_{m}$ be the probability that $\Delta_{e} \geq m$ where $e \in[n]^{2}$ is a random edge. We have

$$
\begin{gathered}
E\left[\Delta_{a}\right]=\sum_{m \geq 1} \operatorname{Pr}\left(\Delta_{e_{1}} \geq m \text { and } \Delta_{f_{1}} \geq m\right)=\sum_{m \geq 1} p_{m}^{2}, \\
E\left[\Delta_{b}\right] \leq \sum_{m \geq 1} \operatorname{Pr}\left(\Delta_{e_{2}} \geq m \text { or } \Delta_{f_{2}} \geq m\right)=\sum_{m \geq 1}\left(2 p_{m}-p_{m}^{2}\right) .
\end{gathered}
$$

Note that $E\left[X\left(G^{+}\right)-X(G)\right]$ may be larger than $\left(E\left[\Delta_{a}\right]+E\left[\Delta_{b}\right]\right) / n$, as we must account for the possibility that $a$ and $b$ intersect a common component of $G$. When this happens the additional contribution to $E\left[X\left(G^{+}\right)-X(G)\right]$ is $2 \cdot\left|C_{a}\right| \cdot\left|C_{b}\right| / n$ where $C_{a}$ is the component of $G$ that intersects $a$ but not $b$ and $C_{b}$ is the component of $G$ that intersects $b$ but not $a$. If we fix the components $C_{a}$ and $C_{b}$ there are 4 vertices among the edges $e_{1}, f_{1}$ that could fall in $C_{a}$ and 4 vertices among the edges $e_{2}, f_{2}$ that could fall in $C_{b}$. Therefore, applying (2.1) we have

$$
\begin{aligned}
E\left[X\left(G^{+}\right)-X(G)\right] \leq & \frac{E\left[\Delta_{a}\right]}{n}+\frac{E\left[\Delta_{b}\right]}{n} \\
& \quad+16 \sum_{i \neq j}\left[\frac{\left|C_{i}\right|}{n}\left(\sum_{k} \frac{\left|C_{k}\right|^{2}}{n^{2}}\right) \frac{\left|C_{j}\right|}{n}\right] \frac{2 \cdot\left|C_{i}\right| \cdot\left|C_{j}\right|}{n} \\
& \leq \frac{1}{n} \sum_{m \geq 1}\left(p_{m}^{2}+2 p_{m}-p_{m}^{2}\right)+\frac{32}{n^{2}} \sum_{k} \frac{\left|C_{k}\right|^{2}}{n} \sum_{i, j} \frac{\left|C_{i}\right|^{2}\left|C_{j}\right|^{2}}{n^{2}} \\
& =\frac{2}{n} \sum_{m \geq 1} p_{m}+\frac{32 X^{3}(G)}{n^{2}} \\
& \leq \frac{4}{n}\left(\frac{1}{n} \sum_{i=1}^{r}\left|C_{i}\right|^{2}\right)^{2}+\frac{32 X^{3}(G)}{n^{2}} .
\end{aligned}
$$

Note that we apply (2.1), dropping the sum of the fourth powers of the component sizes (as we are establishing an upper bound).

Lemma 3.2. Let $G$ be a fixed graph on vertex set $[n]$ with components $C_{1}, \ldots, C_{r}$. Let $e_{1}, f_{1}, e_{2}, f_{2} \in[n]^{2}$ be independent random edges. If $G^{+}=G+\{a, b\}$ where 
$a \in\left\{e_{1}, f_{1}\right\}$ is chosen according to Creator's product rule and $b \in\left\{e_{2}, f_{2}\right\}$ is chosen arbitrarily, then

$$
E\left[X\left(G^{+}\right)\right] \geq X(G)+\frac{4}{n} X^{2}(G)-\frac{4}{n^{3}} \sum_{i=1}^{r}\left|C_{i}\right|^{4}-\frac{16}{n^{5}}\left(\sum_{i=1}^{r}\left|C_{i}\right|^{3}\right)^{2} .
$$

Proof. We follow the proof of Lemma 3.1, noting that here we must account for the possibility that edges $a$ and $b$ join the same pair of components from $G$. The probability that one of the edges $e_{1}, f_{1}$ joins components $C_{i}$ and $C_{j}$ is at most $\frac{4\left|C_{i}\right|\left|C_{j}\right|}{n^{2}}$. Therefore, we have

$$
E\left[X\left(G^{+}\right)-X(G)\right] \geq \frac{E\left[\Delta_{a}\right]}{n}+\frac{E\left[\Delta_{b}\right]}{n}-\sum_{i<j}\left(\frac{4\left|C_{i}\right|\left|C_{j}\right|}{n^{2}}\right)^{2} \frac{2\left|C_{i}\right|\left|C_{j}\right|}{n} .
$$

Now we apply (2.1) to achieve the stated bound, noting that here we cannot drop the sum of fourth powers of the component sizes, as we are establishing a lower bound.

We are now ready to apply the so-called differential equations method.

3.1. Destroyer. Let $c<1 / 2$ be a constant and assume that Destroyer employs Destroyer's product rule. We start by defining some constants. Let $\beta$ be a constant such that

$$
c+\beta<\frac{1}{2} .
$$

Let $\eta$ be a constant such that

$$
4 \eta f(c+\beta)=\frac{4 \eta}{1-2(c+\beta)}<1 .
$$

Let $0=c_{0}<c_{1}<c_{2}<\cdots<c_{N}=c$ be constants that satisfy

$$
\left|c_{i+1}-c_{i}\right|<\eta \quad \text { for } i=0, \ldots, N-1 .
$$

We view these $c_{i}$ 's as landmarks in the process, and we show that whp at each of these landmarks our graph is very well behaved. To be precise, we will show that whp we have

(i) $G_{c_{i} n}$ has a $K_{i}, \alpha_{i}$ component tail (where $K_{i}$ and $\alpha_{i}$ are constants), and

(ii) $X\left(G_{c_{i} n}\right) \leq f\left(c_{i}+\frac{i \beta}{N}\right)=\frac{1}{1-2\left(c_{i}+\frac{i \beta}{N}\right)}$

for $i=1, \ldots, N$. To establish these conditions, we go by induction on $i$.

Assume $G_{c_{i} n}$ satisfies (i) and (ii). First, we establish (i) for $G_{c_{i+1} n}$. Let $H$ be the graph on vertex set $[n]$ with edge set

$$
\left\{e_{k}: c_{i} n<k \leq c_{i+1} n\right\} \cup\left\{f_{k}: c_{i} n<k \leq c_{i+1} n\right\} .
$$

Since $G_{c_{i} n}$ satisfies (ii), we have (applying (3.2))

$$
X\left(G_{c_{i} n}\right) 4 \eta \leq 4 \eta f\left(c_{i}+\frac{i \beta}{N}\right) \leq 4 \eta f(c+\beta)<1 .
$$

It then follows from part (a) of Theorem 2.1 that there exist constants $K_{i+1}, \alpha_{i+1}$ such that whp $G_{c_{i} n}+G_{n, 4 \eta / n}$ has a $K_{i+1}, \alpha_{i+1}$ component tail. Since, using standard techniques, the number of edges in $G_{n, 4 \eta / n}$ is concentrated around $2 \eta n$ and we have (3.3), it follows that $G_{c_{i} n}+H$ has a $K_{i+1}, \alpha_{i+1}$ component tail whp. 
Since $G_{c_{i+1} n}$ is contained in $G_{c_{i} n}+H$, the graph $G_{c_{i+1} n}$ also has a $K_{i+1}, \alpha_{i+1}$ component tail whp.

The proof that $G_{c_{i+1} n}$ satisfies (ii) is more delicate and requires the assumption that $G_{c_{i+1} n}$ satisfies (i) whp (as we have already established). We apply the socalled differential equations method for random graph process to the susceptibility (for an excellent reference for this method see [8]). Set $K=\frac{c_{i+1} n-c_{i} n}{2}$. For $k=$ $0, \ldots, K$ set

$$
X_{k}=X\left(G_{c_{i} n+2 k}\right)
$$

For $k=0, \ldots, K$ set

$$
Y_{k}=X_{k}-f\left(c_{i}+\frac{(i+1) \beta}{N}+\frac{2 k}{n}\right) .
$$

We have

$$
Y_{0}=X_{0}-f\left(c_{i}+\frac{(i+1) \beta}{N}\right) \leq f\left(c_{i}+\frac{i \beta}{N}\right)-f\left(c_{i}+\frac{(i+1) \beta}{N}\right)<-\gamma<0 .
$$

(Note that this inequality defines $\gamma$.) Our aim is to show that $\left\{Y_{i}\right\}$ is a supermartingale with bounded differences. To achieve this, we apply the standard technique of introducing a stopping time. Let $T$ be the smallest index $k$ such that

- $G_{c_{i} n+2 k}$ has a component with more than $\alpha^{\prime} \log n$ vertices (where $\alpha^{\prime} \alpha_{i+1}>$ 1 ), or

- $Y_{k} \geq-\gamma / 2$.

Finally, let $Z_{k}=Y_{\min \{k, T\}}$.

Lemma 3.3. If $n$ is sufficiently large, then the sequence $\left\{Z_{i}\right\}$ is a supermartingale.

Proof. If $k \geq T$, then we (deterministically) have $Z_{k+1}=Z_{k}$.

Suppose $k<T$. It follows that $Y_{k}<-\gamma / 2$. Let $\mathcal{F}_{k}$ be the filtration of our probability space given by the set of edges generated through the first $k$ turns of the game. Applying Lemma 3.1 and the convexity of $f$ we have

$$
\begin{aligned}
E\left[Z_{k+1}-Z_{k} \mid \mathcal{F}_{k}\right]= & E\left[Y_{k+1}-Y_{k} \mid \mathcal{F}_{k}\right] \\
= & E\left[X_{k+1}-X_{k} \mid \mathcal{F}_{k}\right] \\
& -\left[f\left(c_{i}+\frac{(i+1) \beta}{N}+\frac{2 k+2}{n}\right)-f\left(c_{i}+\frac{(i+1) \beta}{N}+\frac{2 k}{n}\right)\right] \\
\leq & \frac{4}{n} X_{k}^{2}+\frac{32}{n^{2}} X_{k}^{3}-\frac{2}{n} f^{\prime}\left(c_{i}+\frac{(i+1) \beta}{N}+\frac{2 k}{n}\right) \\
\leq & \frac{4}{n}\left[f\left(c_{i}+\frac{(i+1) \beta}{N}+\frac{2 k}{n}\right)-\frac{\gamma}{2}\right]^{2}+\frac{32}{n^{2}} X_{k}^{3} \\
& \quad-\frac{2}{n} f^{\prime}\left(c_{i}+\frac{(i+1) \beta}{N}+\frac{2 k}{n}\right) \\
= & \frac{1}{n}\left[\gamma^{2}+\frac{32}{n} X_{k}^{3}-4 \gamma f\left(c_{i}+\frac{(i+1) \beta}{N}+\frac{2 k}{n}\right)\right] \\
\leq & 0
\end{aligned}
$$

for $n$ sufficiently large. 
Lemma 3.4. For $k=0, \ldots, K$ we have

$$
\left|Z_{k+1}-Z_{k}\right| \leq \frac{6\left(\alpha^{\prime}\right)^{2}(\log n)^{2}}{n} .
$$

Proof. If $k \geq T$, then we trivially have $Z_{k+1}=Z_{k}$. If $k<T$, then the largest components in $G_{c_{i} n+2 k}$ have at most $\alpha^{\prime} \log n$ vertices. The maximum change in the susceptibility when we add two edges to such a graph is $6\left(\alpha^{\prime}\right)^{2} \log ^{2} n / n$.

We have

$$
\begin{aligned}
& \operatorname{Pr}\left(X\left(G_{c_{i+1} n}\right)>f\left(c_{i+1}+\frac{(i+1) \beta}{N}\right)\right) \leq \operatorname{Pr}(T \leq K) \\
\leq & \operatorname{Pr}\left(Z_{K} \geq-\gamma / 2\right)+\operatorname{Pr}\left(G_{c_{i+1} n} \text { does not have a } K_{i+1}, \alpha_{i+1} \text { component tail }\right) .
\end{aligned}
$$

The second probability here is $o(1)$ as we noted above. We apply an AzumaHoeffding type inequality (see for example Lemma 4.2 of [8]) to bound the first probability (using Lemmas 3.3 and 3.4). To be precise,

$$
\operatorname{Pr}\left(Z_{K}-Z_{0} \geq \gamma / 2\right) \leq \exp \left\{-\frac{\gamma^{2} n}{72\left(\alpha^{\prime}\right)^{4}(\log n)^{4}}\right\} .
$$

Thus (ii) holds whp.

3.2. Creator. Here we follow the analysis of the previous section to get to a point where the susceptibility is large, and then we apply Theorem 2.1, part (b).

Let $c>1 / 2$ be a constant. Let $\nu=c-1 / 2$. Choose $\tau$ such that

$$
f(\tau) \nu \frac{e^{-8}}{4}>1 \text {. }
$$

Let $\beta>0$ be a constant such that $\tau+\beta<1 / 2$. Choose $\eta$ such that

$$
4 \eta f(\tau+\beta)<1 \text {. }
$$

Let $0=c_{0}<c_{1}<c_{2}<\cdots<c_{N}=\tau+\beta$ be constants such that

$$
\left|c_{i+1}-c_{i}\right| \leq \eta \quad \text { for } \quad i=0, \ldots, N-1 .
$$

Now we define a sequence of times in our process. For $i=0, \ldots, N$ let $T_{i}$ be the smallest value of $k$ for which

$$
X\left(G_{k}\right) \geq f\left(c_{i}-\frac{i \beta}{N}\right) .
$$

We show that whp we have

(i) $G_{T_{i}}$ has a $K_{i}, \alpha_{i}$ component tail (where $K_{i}$ and $\alpha_{i}$ are constants), and

(ii) $T_{i} \leq c_{i} n$

for $i=1, \ldots, N$.

We go by induction on $i$ as in the previous subsection. Suppose $T_{i}$ satisfies conditions (i) and (ii). Note that condition (i) and the definition of $T_{i}$ (given in (3.6) ) imply

$$
X\left(G_{T_{i}}\right)=f\left(c_{i}-\frac{i \beta}{N}\right)+o(1) .
$$

We consider the $2 K=\left(c_{i+1}-c_{i}\right) n$ turns that follow turn $T_{i}$. As there are less than $2 \eta n$ random edges generated during these turns, it follows from (3.5) and part (a) of 
Theorem 2.1 that whp $G_{T_{i}+2 K}$ has a $K_{i+1}, \alpha_{i+1}$ component tail for some constants $K_{i+1}, \alpha_{i+1}$. For $k=0, \ldots, K$ set

$$
Y_{k}=X\left(G_{T_{i}+2 k}\right)-f\left(c_{i}-\frac{(i+1) \beta}{N}+\frac{2 k}{n}\right) .
$$

Note that $Y_{0}>\gamma$ for a constant $\gamma>0$. Let the stopping time $T$ be the smallest index $k$ such that $G_{T_{i}+2 k}$ has a component with more than $\alpha^{\prime} \log n$ vertices or $Y_{k}<\gamma / 2$. This yields a submartingale with bounded differences. By an application of the Azuma-Hoeffding inequality, whp we have

$$
X\left(G_{T_{i}+2 K}\right)>f\left(c_{i+1}-\frac{(i+1) \beta}{N}\right) .
$$

Conditions (i) and (ii) on $T_{i+1}$ follow.

Now suppose $T_{N}$ satisfies conditions (i) and (ii). The graph $G_{T_{N}}$ has a $K_{N}, \alpha_{N}$ component tail and $T_{N} / n<c_{N}=\tau+\beta$. We consider the $\nu n$ turns after turn $T_{N}$. For Creator's turn $k$ during this interval, the edge chosen is a purely random edge whenever $e_{k}$ is isolated. (This assertion would follow from the assumption that Creator's product rule imposes the tie-breaking convention that $f_{k}$ is chosen when $e_{k}$ is isolated. Alternately, we could focus only on rounds where one edge is isolated and the other is not. For the sake of brevity we omit this detail). The number of vertices that do not appear in the set of edges

$$
\left\{e_{k}: 1 \leq k \leq n\right\} \cup\left\{f_{k}: 1 \leq k \leq n\right\}
$$

is concentrated around $n e^{-4}$. We conclude that during the $\nu n$ turns after turn $T_{N}$ whp at least $e^{-8} \nu n / 4$ purely random edges are added to the graph $G$. It follows from Theorem 2.1 part (b) and (3.4) that $G_{T_{N}+\nu n}$ has a component with $\Omega(n)$ vertices whp.

\section{Product Rule wins (proof of Theorem 1.2)}

In Section 3.1 we saw that, under the assumption that Destroyer uses Destroyer's product rule, the susceptibility is bounded above by the trajectory $f$ that describes the evolution of the susceptibility for the Erdős-Rényi random graph. If we add the assumption that Creator makes many mistakes (relative to the product rule), then we can adapt our argument to show that there are constants $c<1 / 2$ and $\delta>0$ such that whp the susceptibility of $G_{c n}$ is bounded by $f(c-\delta)$. Since the argument that bounds the progress of the susceptibility does not depend on the number of edges in the graph, any ground that Creator loses (relative to $f$ ) cannot be regained. This gives the proof of part (a) of Theorem 1.2. We now sketch the details. The proof of part (b) of Theorem 1.2 follows along similar lines.

Let $\delta, \beta>0$ be constants such that

$$
f\left(\frac{1}{2}-\frac{\epsilon}{2}-\delta\right)=f\left(\frac{1}{2}-\frac{\epsilon}{2}+\beta\right)-\frac{\epsilon}{2} .
$$

Let $\eta>0$ be chosen such that

$$
4 \eta f\left(\frac{1}{2}-\frac{\epsilon}{2}+\beta\right)<1 .
$$

Let $0=c_{0}<c_{1}<c_{2}<\cdots<c_{N}=1 / 2-\epsilon / 2$ be constants that satisfy

$$
\left|c_{i+1}-c_{i}\right|<\eta \quad \text { for } i=0, \ldots, N-1 .
$$


We follow the argument of Section 3.1 to show that the susceptibility is bounded at each of the landmarks. However, instead of working with $X_{k}=X\left(G_{c_{i} n+2 k}\right)$, we consider

$$
X_{k}^{\prime}=X\left(G_{c_{i} n+2 k}\right)+\frac{A_{c_{i} n+2 k}}{n} .
$$

If we consider two turns of the game and condition on the event that Creator makes a mistake relative to the product rule, then the expected change in the susceptibility is bounded above by the expression given in Lemma 3.1 minus $2 / n$. It follows that we can apply the argument in Section 3.1 directly to $X^{\prime}$ to show that we have

$$
X^{\prime}\left(G_{c_{i} n}\right)<f\left(c_{i}+\frac{\beta i}{N}\right) \quad \text { for } \quad i=1, \ldots, N .
$$

So, we have

$$
\begin{aligned}
& X\left(G_{n / 2-\epsilon n / 2}\right)=X\left(G_{c_{N} n}\right)=X^{\prime}\left(G_{c_{N} n}\right)-\frac{A_{c_{N} n}}{n} \leq f\left(c_{N}+\beta\right)-\frac{\epsilon}{2} \\
&=f\left(\frac{1}{2}-\frac{\epsilon}{2}+\beta\right)-\frac{\epsilon}{2}=f\left(\frac{1}{2}-\frac{\epsilon}{2}-\delta\right) .
\end{aligned}
$$

Now let $\eta^{\prime}>0$ satisfy

$$
4 \eta^{\prime} f\left(\frac{1}{2}-\frac{\delta}{4}\right)<1
$$

We consider landmarks $c_{N}<c_{N+1}<c_{N+2}<\cdots<c_{M}=1 / 2+\delta / 4$ which satisfy

$$
\left|c_{i+1}-c_{i}\right|<\eta^{\prime} \quad \text { for } i=N, \ldots, M-1 .
$$

Following the machinery of Section 3.1 we see that $G_{c_{i} n}$ has a $K_{i}, \alpha_{i}$ component tail and

$$
X\left(G_{c_{i} n}\right)<f\left(c_{i}-\delta+\frac{\delta(i-N)}{2(M-N)}\right)
$$

for $i=N+1, \ldots, M$. This completes our sketch of the proof of part (a) of Theorem 1.2

\section{REFERENCES}

[1] T. Bohman and A. Frieze, Avoiding a giant component. Random Structures and Algorithms 19 (2001), 75-85. MR.1848028 (2002g:05169)

[2] T. Bohman, A. Frieze and N. Wormald, Avoidance of a giant component in half the edge set of a random graph. Random Structures and Algorithms 25 (2004) 432-449. MR2099213 (2005f:05151)

[3] T. Bohman and J. H. Kim, A phase transition for avoiding a giant component. Random Structures and Algorithms 28 (2006) 195-214. MR2198497

[4] T. Bohman and D. Kravitz, Creating a giant component. Combinatorics, Probability and Computing 15 (2006) 489-511. MR2238042

[5] A. Flaxman, D. Gamarnik and G. Sorkin, Embracing the giant component. pp. 69-79 in Latin 2004: Theoretical Informatics, Farach and Coltin eds. Springer 2004. MR2095182

[6] J. Spencer, Percolating thoughts. Unpublished manuscript dated January 31, 2001.

[7] J. Spencer and N. Wormald, Birth Control for Giants. Combinatorica, to appear.

[8] N. Wormald, The differential equations method for random graph processes and greedy algorithms. pp. 73-155 in Lectures on Approximation and Randomized Algorithms, Karoński and Prömel eds. PWN, Warsaw 1999. 
Department of Mathematical Sciences, Carnegie Mellon University, Pittsburgh, Pennsylvannia 15213

E-mail address: ajbever@andrew.cmu.edu

Department of Mathematical Sciences, Carnegie Mellon University, Pittsburgh, PenNSYlVANNIA 15213

E-mail address: tbohman@math.cmu.edu

Department of Mathematical Sciences, Carnegie Mellon University, Pittsburgh, Pennsylvannia 15213

E-mail address: alan@random.math.cmu.edu

Department of Mathematical Sciences, Carnegie Mellon University, Pittsburgh, Pennsylvannia 15213

E-mail address: pikhurko@andrew.cmu.edu 